\title{
Mise en place et fonctionnement des feuilles de colza d'hiver : relations azote-carbone et sénes- cence
}

\author{
Anne-Marie TRIBOI-BLONDEL \\ avec la collaboration technique de Joëlle Messaoud et Paul Rousseau \\ I.N.R.A., Station d'Agronomie, 12, avenue du Brézet, F 63039 Clermont-Ferrand Cedex
}

RÉSUMÉ

\begin{abstract}
Chez le colza d'hiver, Brassica napus L. oleifera Metzg., variété Jet Neuf, nous avons suivi au cours d'un cycle complet de végétation au champ, le rythme d'apparition et de disparition des différentes feuilles et l'évolution de leurs poids, surfaces, teneurs en azote et glucides solubles.

Le thermochrone (en degrés-jours) diminue fortement des feuilles dautomne-hiver $\left(75^{\circ} \mathrm{J}\right)$ aux feuilles de printemps $\left(20^{\circ} \mathrm{J}\right)$.

La teneur en glucides solubles est peu variable sur la plante avec le rang des feuilles; elle varie essentiellement selon la saison: augmentation à l'automne, maximum en janvier (jusqu'à 25 p. 100), diminution progressive au printemps en période de croissance active.

La teneur en azote total, au contraire, varie essentiellement avec l'âge de la feuille : 6-7 p. 100 lors de l'émission et $2-2,5$ p. 100 à la chute.

Cette teneur de 2 p. 100 semble caractériser la sénescence de la feuille.
\end{abstract}

Mots clés additionnels: Phyllochrone, thermochrone, masse surfacique, glucides, source-puits.

\begin{abstract}
Emergence and function of winter rape leaves : nitrogen-carbon relationships and senescence.
Winter rapeseed, Brassica napus L. oleifera Metzg. cv. Jet Neuf, in field was studied over its whole development period in the field. Emergence and disappearance rates of leaves were examined, together with their weights, areas, nitrogen and water-soluble carbohydrate concentrations. The thermochrone (in degree-days) decreased strongly from autumn-winter leaves $\left(75^{\circ} \mathrm{D}\right)$ to spring leaves $\left(20^{\circ} \mathrm{D}\right)$. At time $\mathrm{t}$, water-soluble carbohydrate concentration was about constant in the different leaves; it varied essentially with the season : rise in autumn, maximum value in January $(25 \%)$ and progressive fall in spring, during the active growth period. In contrast, total nitrogen concentration varied essentially with leaf age: 6-7\% at emergence and $2-2.5 \%$ at disappearance. This concentration of $2 \%$ seems to characterize the senescence of the leaf.
\end{abstract}

Additional key words : Phyllochrone, thermochrone, specific leaf weight, carbohydrates, source-sink.

\section{INTRODUCTION}

On explique assez bien la production de matière sèche d'une culture par le rayonnement intercepté (GOSSE et al., 1983).

Le suivi de l'assimilation nette de différents peuplements de colza d'hiver, nous a conduit à écrire (RobeliN \& TRIBOÏ, 1983) que «tout se passe comme si » la photosynthèse s'ajustait à la demande imposée par le déroulement de croissance, lui-même conditionné par l'interaction génotype-milieu (température, photopé- riode) et les ressources mobilisables antérieurement stockées.

Notre étude est réalisée aujourd'hui à un niveau plus fin dans le couvert. Elle vise la mise en place et le fonctionnement des feuilles : principaux capteurs photosynthétiques chez le colza (les siliques en prenant le relais en fin de cycle).

D'après MAJOR et al., 1980, pour augmenter les rendements du colza, il faudrait prolonger la durée de vie des feuilles et les principales causes de nécrose 
précoce seraient une carence en azote, un stress hydrique ou un déséquilibre source (feuilles) — puits (fleurs et siliques). RoOD et al., 1984, trouvent de plus une relation entre la sénescence des feuilles, siliques et tiges et l'arrêt de l'absorption des nitrates.

Cet article a pour but essentiel l'étude du déterminisme de la chute des feuilles précisément en relation avec la teneur en azote.

\section{MATÉRIEL ET MÉTHODES}

\section{A. Matériel : Brassica napus L. Oleifera Metzg., variété Jet Neuf}

L'étude a été réalisée sur des plantes prélevées dans une parcelle du domaine de Crouelle (I.N.R.A., Clermont-Ferrand) en sol argilo-calcaire profond décrit par GACHON (1973).

La conduite de la culture, à savoir, préparation du sol, semis (le 7 septembre 1984), fertilisation, désherbage, lutte contre insectes et maladies, et la récolte ont été réalisées selon un itinéraire technique classique.

Précisons toutefois que la densité moyenne à la reprise de végétation était de 70 plantes au $\mathrm{m}^{2}$, l'écartement entre lignes de $20 \mathrm{~cm}$, et la fertilisation azotée de 50 unités au semis et 150 unités appliquées en 2 fois. en février et mars sous forme d'ammonitrate.

Le rendement en grains à la récolte, le 22 juillet 1985 , était de $40 \mathrm{q} \mathrm{ha}^{-1}$ (à 0 p. 100 d'humidité).

\section{B. Echantillonnage}

Des prélèvements de plantes ont été effectués toutes les 3 semaines environ sauf en janvier (période de froid exceptionnel en 1985).

Les surfaces de prélèvement, choisies en fonction de la régularité des plantes sur la ligne ont varié de 1 à $2,5 \mathrm{~m}^{2}$ soit 70 à 200 plantes, selon les dates et le développement des plantes. Pour chaque plante, après lavage des racines, on procède à la séparation en différents compartiments. Les feuilles sont détachées puis classées par lots suivant leur ordre d'insertion sur la tige. Seules les feuilles de l'axe principal ont été étudiées. La numérotation s'effectue à partir de la feuille $n^{\circ} 1$ située au-dessus des cotylédons et ainsi de suite jusqu'à l'apex. Lorsqu'une feuille tombe, elle laisse sur la tige une cicatrice qui permet de retrouver à chaque prélèvement le nombre de feuilles tombées et les numéros d'ordre des feuilles présentes.

Pour chaque date de prélèvement on peut ainsi connaître le pourcentage de feuilles émises et tombées pour chaque niveau. A partir de ces données nous avons pu déterminer avec précision la date d'émergence et de chute de chaque feuille, date correspondant à 50 p. 100 de l'effectif.

En réalité, le rythme de disparition des feuilles a été suivi de manière plus précise, par récolte individuelle de chaque feuille tombée (tous les 3-4 jours) après repérage du niveau sur chaque plante d'une microparcelle de $1 \mathrm{~m}^{2}$, tout au long du cycle de développement.
On a également calculé les sommes de température (base 0 ), en degrés jours : ment,

- à partir de la levée, pour chaque date de prélève- à partir de la date d'émergence, pour chaque feuille.

\section{Analyses}

Nous avons déterminé pour chaque feuille, séparée en limbe et pétiole :

- la matière fraîche et sèche (après passage à l'étuve à $80^{\circ} \mathrm{C}$ ),

- la surface foliaire $\left(\mathrm{en} \mathrm{cm}^{2}\right.$ ) au planimètre optique AAM-5, HAYASHI DENKO CO-LTD,

- la teneur en azote total avec un analyseur KJELTEC AUTO 1030, TECATOR,

- les sucres solubles totaux de fragments de limbes décongelés, après extraction dans un mélange éthanoleau (Besle \& Pitiot, 1976 ; Jourdan, 1980), purification sur charbon végétal activé, et dosage à l'anthronesulfurique.

\section{RÉSULTATS}

\section{A. Rythme d'émergence et de chute des feuilles — durée de vie}

L'intervalle de temps entre les dates d'émergence de 2 feuilles successives est appelé phyllochrone. Durée de vie d'une feuille et phyllochrone sont exprimés en temps réel (jours) ou en degrés jours. Le phyllochrone exprimé en degrés jours peut être appelé thermochrone (MALVOISIN, 1984).

\section{Phyllochrone (fig. 1a)}

A l'automne (du semis au 1/11) et à la reprise de végétation (à partir du 15/03), le rythme d'émergence est rapide et assez comparable : le phyllochrone varie de 1,5 à 3 jours. Pendant l'hiver (du $1 / 11$ au 15/03), au contraire, le rythme est lent et le phyllochrone passe à 16 jours en moyenne pour les 8 feuilles apparues.

Le rythme de disparition des feuilles est parallèle au rythme d'émergence avec contrairement à ce qu'on aurait pu imaginer un ralentissement considérable lorsque les températures baissent.

La durée de vie est d'autant plus longue ( 5 mois pour la feuille $8 ; 1$ mois pour la feuille 2 ) que la température est basse.

\section{Thermochrone (fig. 1b)}

Le thermochrone (exprimé en degrés jours) est, en période automno-hivernale, plus régulier que le phyllochrone : 75 degrés jours en moyenne pour les 16 premières feuilles. Mais on observe ensuite un changement de pente : le thermochrone passe à 20 degrés jours pour les feuilles supérieures (17 à 33). 

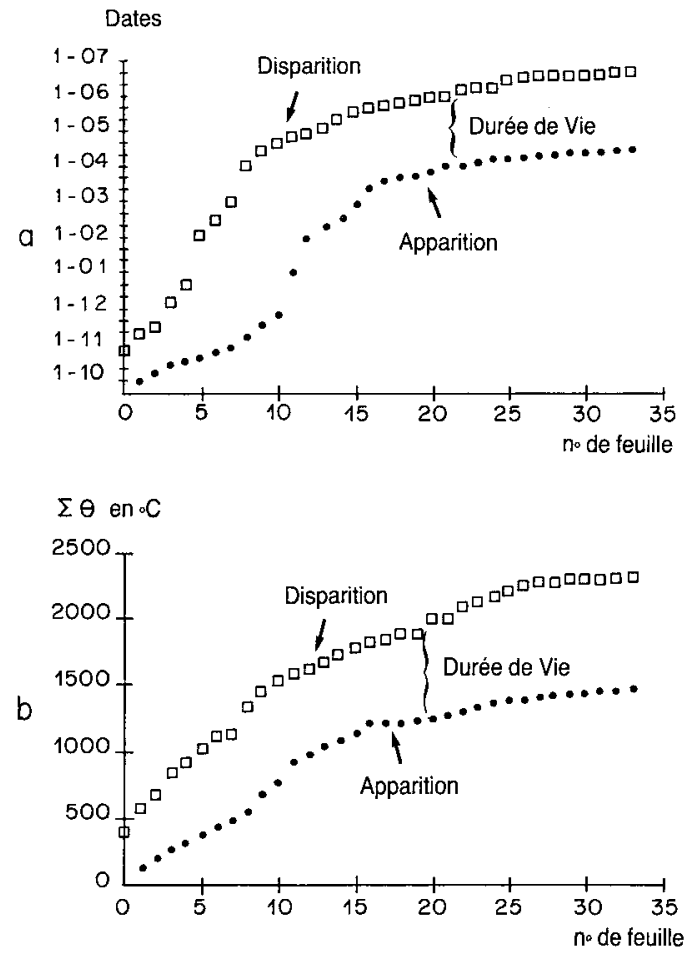

Figure 1

Rythme d'apparition et de disparition des feuilles, et durée de vie des feuilles:

a) en fonction du temps (j).

b) en fonction des sommes de températures $\left({ }^{\circ} \mathrm{Cj}\right.$, base $\left.0^{\circ} \mathrm{C}\right)$.

Rate of leaf emergence and disappearance, and lifespan of leaves : a) with time (days),

b) with accumulated temperature $\left({ }^{\circ} \mathrm{Cd}\right.$, base $\left.0^{\circ} \mathrm{C}\right)$

Le rythme de disparition des feuilles (exprimé en degrés jours) est assez comparable au rythme d'émergence.

La durée de vie, exprimée en degrés jours $\left({ }^{\circ} \mathrm{J}\right)$ varie de 440 pour la feuille 1 , à 850 pour les feuilles $26-33$, avec une augmentation assez régulière dans l'intervalle 2-25.

\section{B. Forme et taille des feuilles}

A chaque date de prélèvement, nous avons effectué des photocopies des différentes feuilles d'une plante d'un poids de matière fraîche moyen; nous avons ensuite sélectionné la feuille de taille maximale pour chaque rang $(1,2, \ldots 33)$. Différents types peuvent être observés (fig. 2) :

- les feuilles automnales ( 1 à 5), dont la taille définitive du limbe, crôit de 24 (1) à $91 \mathrm{~cm}^{2}$ (5) ; le limbe est large mais de plus en plus lobé et le pétiole important,

- les feuilles hivernales ( 6 à 15), dont la taille définitive se restreint et le limbe devient plus étroit : elles sont plus découpées et proportionnellement moins pétiolées que les précédentes,

- les feuilles printanières (16 et suivantes), émises à la reprise de végétation qui comportent des stipules à la base du pétiole, deviennent moins découpées et se transforment finalement (21) en feuilles lancéolées.

La plus grande feuille apparait donc à l'automne ( 5 ou 6), émergent ensuite des feuilles de taille décroissante.

\section{Masse surfacique. Azote total. Glucides solubles}

Pour chaque feuille et en fonction de la somme des températures moyennes journalières (base $0{ }^{\circ} \mathrm{C}$ ) calculée à partir de la date d'émergence $(50 \mathrm{p} .100$ des feuilles apparues), nous avons déterminé la masse surfacique sèche du limbe (MSS), exprimée en $\mathrm{mg} / \mathrm{cm}^{2}$, la teneur en glucides solubles totaux (GST), exprimée en p. 100 de la matière sèche du limbe et la teneur en azote total (NT), exprimée en p. 100 de la matière sèche de la feuille entière, limbe + pétiole. Certains points de MSS, GST ou NT sont situés sur les courbes avant le 0 de l'abscisse (fig. 3, 4 et 5) car ils correspondent à des feuilles dont l'effectif était inférieur à 50 p. 100 (valeur permettant de déterminer la date d'émergence) lors du prélèvement.

MSS et GST montrent des dynamiques très différentes qui nous ont permis de classer les feuilles en 3 catégories :

\section{Les feuilles 1 à 5 (fig. 3)}

La MSS d'émission de la feuille s'élève avec le rang et reste constante ou augmente légèrement au cours du temps. Le pourcentage de GST évolue assez parallèlement à la MSS pour chaque feuille mais les amplitudes de variations sont plus prononcées : 2 à 25 p. 100 pour les feuilles 4 et 5 , valeur qui les caractérise lors de leur chute.

Les feuilles naissent avec environ 6 p. 100 de NT et disparaissent avec 2 p. 100.

\section{Les feuilles 6 à 13 (fig. 4)}

Comme précédemment la MSS d'émission s'élève et ce jusqu'à la feuille 10 , mais on remarque une nette diminution de la MSS à partir de la première quinzaine d'avril (montaison). Cette diminution apparaît bien évidemment, sur le graphique, à des âges différents (en $\left.{ }^{\circ} J\right)$ selon le rang de la feuille.

Le pourcentage de GST, comme dans les feuilles 4 et 5 augmente dans un premier temps pour les feuilles 6,7 , 8 , mais diminue ensuite et ceci parallèlement à la MSS, début avril et donc à des âges variables selon le rang de la feuille.

Les feuilles naissent avec environ $6 \mathrm{p} .100 \mathrm{de}$ NT et disparaissent avec 2 p. 100. Notons sur le tracé des courbes un pic d'égale importance qui apparaît d'autant plus précocement que la feuille est de rang élevé. Ce pic résulte de l'apport d'engrais azoté le 6 février et le 15 mars qui ne rehausse que très temporairement les teneurs.

\section{Les feuilles 14 à 30 (fig. 5)}

MSS, GST et NT, à leur valeur maximale lors de l'émission de chaque feuille et peu différente avec le rang, diminuent régulièrement pendant la vie de la feuille. Les teneurs en NT évoluent encore entre 6 p. 100 à la naissance et 2 p. 100 à la chute.

\section{DISCUSSION ET CONCLUSION}

\section{A. Mise en place des feuilles}

Le bourgeon terminal de la tige émet des feuilles puis des fleurs, il en est de même pour les bourgeons latéraux 

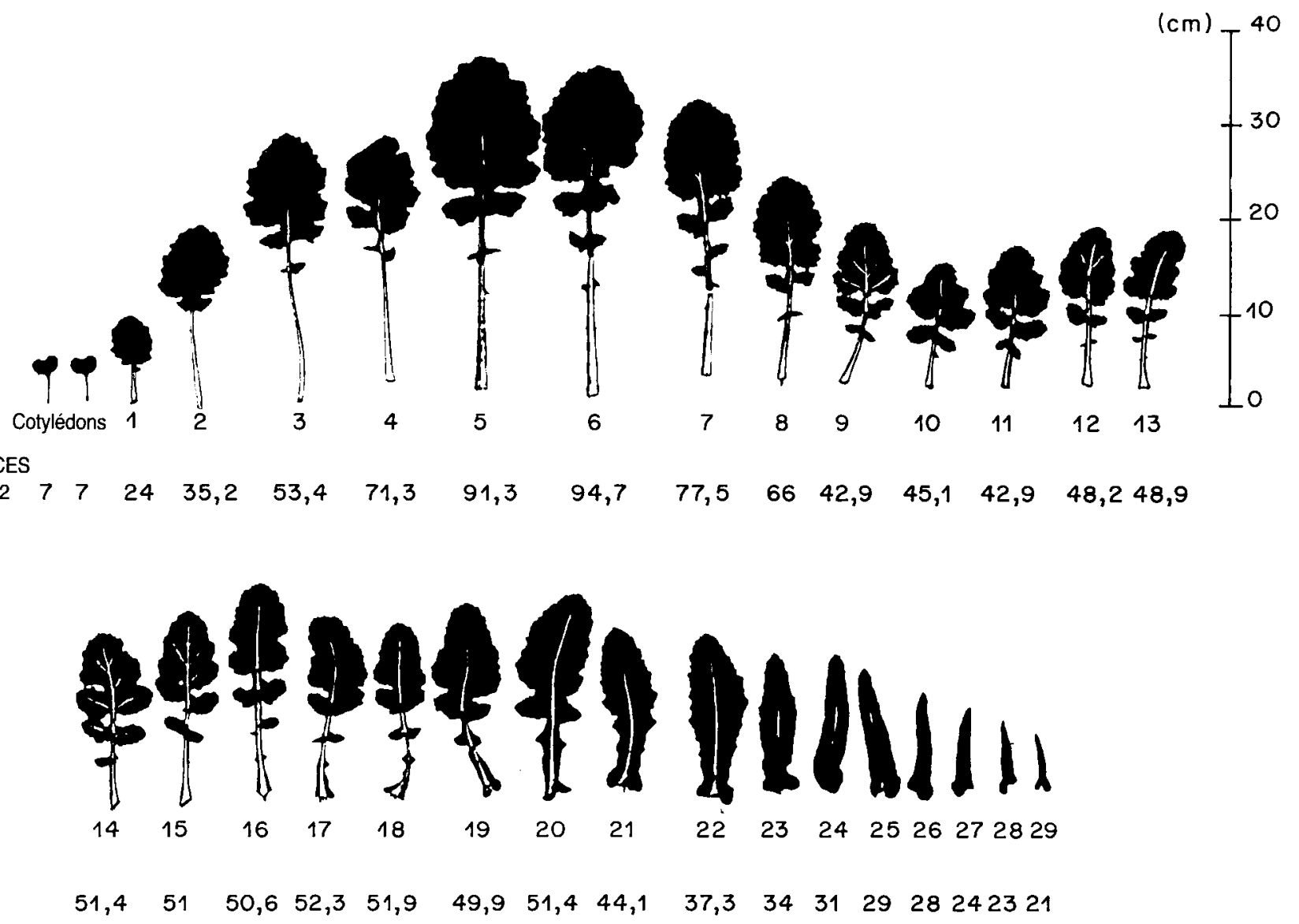

Figure 2

Forme, longueur et surface maximule des feuilles.

Shape, length and maximum arca of leaves.

qui apparaissent à l'aisselle des feuilles de la tige principale et donneront au colza sa forme buissonnante.

Après vernalisation ou induction par le froid (lempérature inférieure ou égale à $17^{\circ} \mathrm{C}$ pour la variété Jet Neuf(Netzer, 1986 et Netzer et al., 1986), l'apex plat, générateur de feuilles se gonfle en dôme et forme des primordia floraux (tubérosités). Les modifications morphologiques du bourgeon lors de cette transformation ont été bien décrites par TiTTONEL et al. (1982). TiTtonel (1987) a démontré de plus que la transformation interne du méristème apical coïncide avec des événements visibles sur la plante. Nous retiendrons parmi eux le changement de rythme d'émergence des feuilles et une modification de la morphologie foliaire (présence de stipules à la base du pétiole).

Dans notre étude nous confirmons les résultats de TitTonel (1987), en ce qui concerne le changement de rythme d'émergence des feuilles, $75^{\circ} \mathrm{J}$ pour les 16 premières feuilles, $20^{\circ} \mathrm{J}$ pour les suivantes et la concordance du point de changement de pente avec la forme des feuilles: présence de stipules sur la feuille 16. On peut par conséquent, bien situer l'induction florale de l'apex au changement de pente du thermochrone. Les feuilles 17 et suivantes sont initiées en "phase préflorale » et changent de forme. A noter également des différences importantes dans le fonctionnement de ces fecilles "végétatives " (1 à 14-15) ou «préflorales » (16 et suivantes).

\section{B. Fonctionnement des feuilles}

A partir de l'émergence et jusqu'à un stade d'expansion du limbe variable selon les espèces, la feuille en croissance est hétérotrophe pour le carbone (DALE, 1982) : elle dépend des métabolites provenant des vieilles feuilles; elle importe.

Elle devient ensuite autotrophe: les chloroplastes s'assemblent, l'activité photosynthétique (par unité de surface) augmente pour être maximale avant que la taille définitive ne soit atteinte (HOPKINSON, 1964 cité par Dale, 1982; Thomas \& Stoddart, 1980). Les exportations d'assimilats deviennent alors importantes.

D'après PATE \& ATKINS (1983), une feuille de lupin blanc au cours de sa vie (66 jours), et pour une structure finale contenant $48 \mathrm{mgC}(\mathrm{Q})$, importe $60 \mathrm{mg} \mathrm{C}$ (I), respire la nuit $52 \mathrm{mg} \mathrm{C}(\mathrm{R})$, fixe le jour (photosynthèse nette) $807 \mathrm{mg} \mathrm{C}\left(\mathrm{P}_{\mathrm{n}}\right)$. On peut donc estimer que globalement les importations compensent le carbone structural final $(Q \simeq I)$, et que les exportations sont environ 13 fois plus élevées que les importations $\left(P_{n}-R / I\right)$. Ces quelques chiffres nous permettent de mieux comprendre la dynamique de fonctionnement d'une feuille.

Le saccharose (Dufrus \& DuFfus, 1984) représente la forme essentielle de transport des assimilats, des sites de synthèse aux sites d'utilisation ou de stockage via le phloème.

La sénescence, définie comme l'ensemble des modifications physiques et métaboliques, conduisant à la mort 

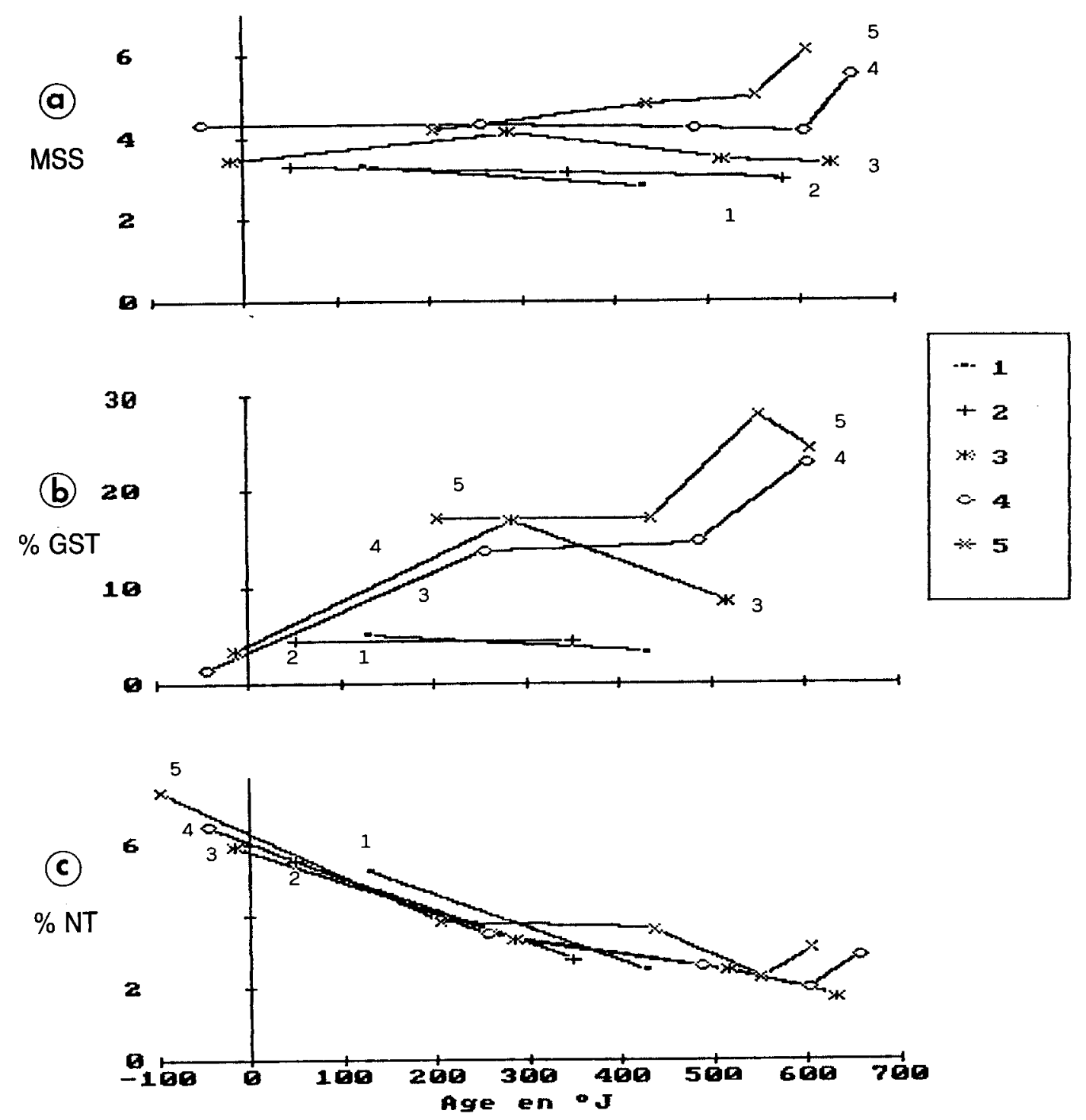

Figure 3

Feuilles 1 à 5 : Evolution, en fonction de l'âge de la feuille, exprimé en température cumulée $\left(\Sigma \theta\right.$, en $\left.{ }^{\circ} \mathrm{Cj}\right)$ de :

(a) la masse surfacique sèche ou MSS, en $\mathrm{mg} \mathrm{cm}^{2}$,

(b) la teneur en glucides solubles totaux ou \% GST

(c) la teneur en azote total ou \% NT.

Leaves 1 to 5 : Changes with increasing leaf age (accumulated temperature, $\Sigma \theta$, in ${ }^{\circ} \mathrm{Cd}$ ) in

(a) specific leaf weight or $M S S$, in $\mathrm{mg} \mathrm{cm}^{-2}$,

(b) watersoluble carbohydrate content or \% GST

(c) nitrogen content or \% NT.

de la feuille (LÉOPOLD, 1961, 1975) correspond d'après THIMANN (1980) à :

- l'activation d'enzymes spécifiques de dégradation de macromolécules comme les acides nucléiques, les pigments, les protéines,

- la transformation de ces produits de dégradation,

- le transfert de ces derniers, dans les jeunes feuilles ou les organes de stockage, essentiellement sous forme d'asparagine et de glutamine (BRAY, 1983).

MAKINO et al. (1984a et $b$ ), montrent qu'une feuille de riz entre 15 et 80 jours, voit ses quantités de RuBP carboxylase et autres protéines solubles nettement diminuer. Il existe une relation entre concentration en azote (N) et en RuBP carboxylase qui représente 28 p. 100 de l'azote de la feuille. La carboxylase agirait comme un composant de stockage d'azote.
Nos résultats démontrent clairement que la feuille est émise avec un pourcentage d'azote très élevé (6-7 p. 100) correspondant vraisemblablement à un équipement fonctionnel (enzymatique) maximal dont l'essentiel est représenté par la RuBP carboxylase. On assiste, au cours de la croissance, à une dilution de l'azote mais la quantité totale ne diminue qu'à la fin de la vie de la feuille. Ceci semblerait indiquer que la sénescence de la feuille, définie comme une diminution des capacités fonctionnelles traduite ici dans l'évolution des teneurs, intervient très tôt.

Toutes les feuilles tombent lorsque la teneur en $N$ est de l'ordre de 2 p. 100 , ce seuil correspondrait pour la feuille à l'azote de structure.

Chez la plupart des plantes, le carbone de l'amidon et des sucres solubles (PATE \& LAYZELL, 1981) représenterait, à la sénescence, un très faible réservoir comparé au 

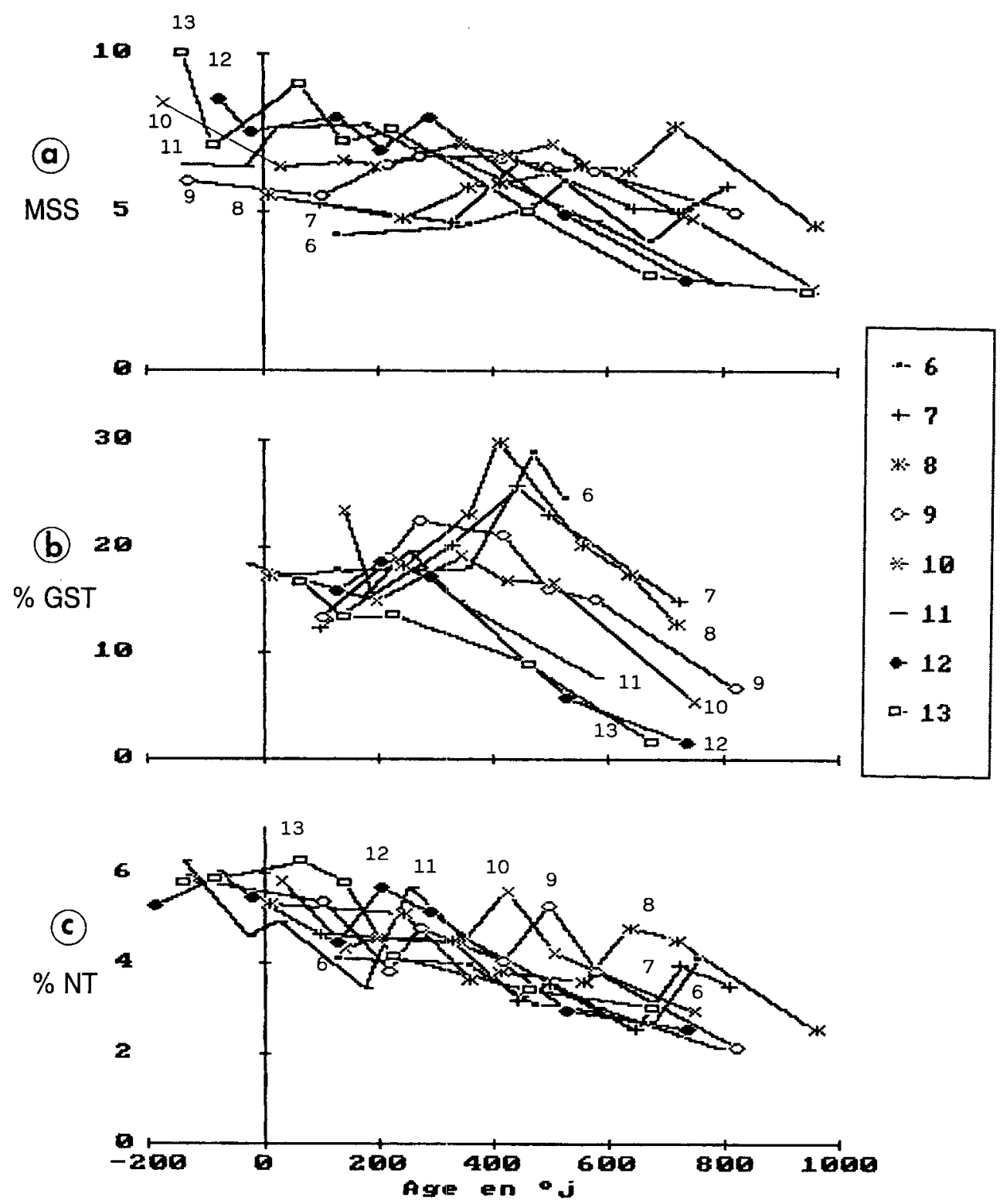

Figure 4

Feuilles 6 à 13 : Evolution, en fonction de l'âge de la feuille, exprimé en lempératuré cumulée ( $\Sigma \theta$, en $\left.{ }^{\circ} \mathrm{Cj}\right)$ de :

(a) la masse surfacique sèche ou $M S S$, en $\mathrm{mg} \mathrm{cm}^{2}$,

(b) la teneur en glucides solubles totaux ou \% GST,

(c) la teneur en azote total ou $\%$ NT.

Leaves 6 to 13 : Changes with increasing leaf age (accumulated temperature, $\Sigma \theta$, in ${ }^{\circ} \mathrm{Cd}$ ) in

(a) specific leaf weight or $M S S$, in $\mathrm{mg} \mathrm{cm}^{-2}$

(b) watersoluble carbohydrate content or \% GST.

(c) nitrogen content or $\% N T$.

carbone de la cellulose et de la lignine (parois des cellules). Cette observation n'est valable que pour les feuilles de type «printemps» puisque les feuilles d'"automne" disparaissent avec plus de 20 p. 100 de sucres solubles. On peut expliquer ce phénomène par un déséquilibre $\mathrm{C} / \mathrm{N}$ dû à l'abaissement des températures à l'automne (Tribol, 1984). Les assimilats en excès par rapport aux besoins instantanés pour la croissance, sont stockés dans les racines (QuILLERE, 1986) mais ces puits de stockage étant de faible taille, les feuilles restent engorgées de glucides jusqu'à leur chute.

A noter que les feuilles les plus grandes (5 et 6) contiennent les plus fortes teneurs en glucides à leur disparition.
Pour les 3 groupes de feuilles, d'automne, d'hiver et de printemps, la durée de vie moyenne est respectivement 540,690 et $760^{\circ} \mathrm{J}$.

Durée de vie (exprimée en degrés-jours) et teneurs en sucres solubles à la chute de la feuille semblent être inversement corrélés. Comment interpréter ce résultat?

Pour certains auteurs (MAJOR et al., 1980 ; THIMANN, 1980; ROBELIN, communication personnelle), la suppression des puits prolonge la vie de la feuille. Pour d'autres, au contraire (NeAles \& Incoll, 1968 ; DaIE, 1985 ; VANN et al., 1986) la présence d'un fruit prolonge la vie de la feuille et une demande accrue du puits stimule la photosynthèse de la source (synergie sourcepuits). A l'inverse, lorsque la demande diminue il y aurait réduction de la fixation de $\mathrm{CO} 2$ par rétroinhibition due à l'accumulation des produits terminaux. 


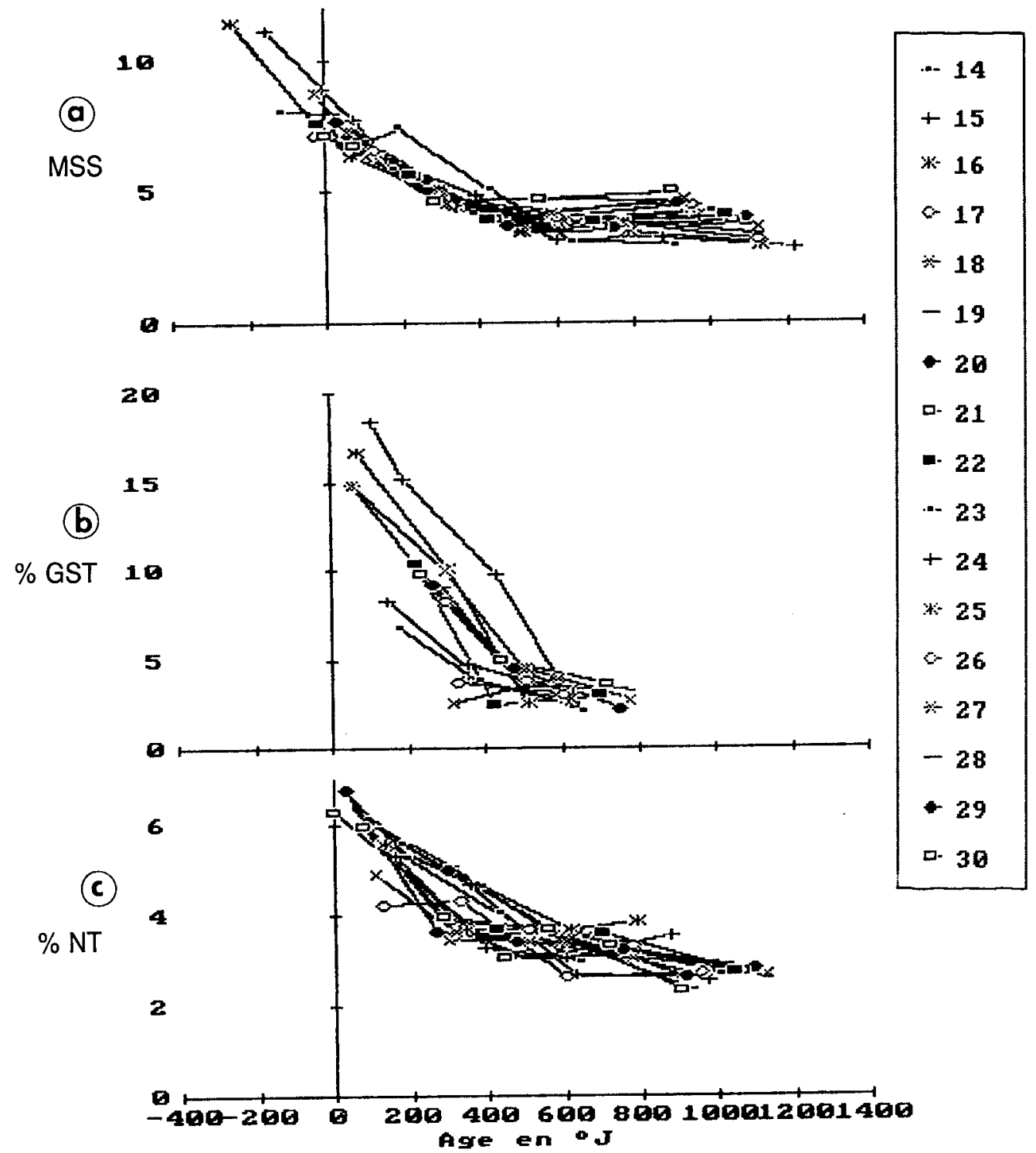

Figure 5

Feuilles 14 à 30 : Evolution, en fonction de l'âge de la feuille, exprimé en température cumulée ( $\Sigma \theta$, en $\left.{ }^{\circ} \mathrm{Cj}\right)$ de (a) la masse surfacique sèche ou MSS, en $\mathrm{mg} \mathrm{cm}^{2}$,

(b) la teneur en glucides solubles totaux ou \% GST,

(c) la teneur en azote total ou \% NT.

Leaves 14 to 30 : Changes with increasing leaf age (accumulated temperature, $\Sigma \theta$, in ${ }^{\circ} \mathrm{Cd}$ ) in .

(a) specific leaf weight or MSS, in $\mathrm{mg} \mathrm{cm}^{-2}$

(b) watersoluble carbohydrate content or \% GST

(c) nitrogen content or $\% N T$

A l'automne les sources sont de taille importante par rapport aux puits limités au méristème apical, aux feuilles en croissance et aux racines. Une fois la croissance assurée et le puits de stockage (racines) rempli, il se peut que l'accumulation de sucres solubles dans les feuilles entraîne une réduction de la photosynthèse. On observe en tout cas une faible durée de vie de ces feuilles.

Au printemps, au contraire, les puits de croissance et de stockage deviennent de plus en plus importants par rapport aux sources qui vraisemblablement fonctionnent de façon intense. Les transferts sont tels que dès l'apparition de la feuille, les teneurs en glucides solubles chutent. La durée de vie de ces feuilles est la plus longue. 


\section{RÉFÉRENCES BIBLIOGRAPHIQUES}

Besle S. M., Pitiot M., 1976. Extraction et purification des glucides : application à divers aliments dérivés du soja. Ann. Biol anim. Biochim. Biophys., 16, 753-772

Bray C. M., 1983. Nitrogen metabolism in plants. Longman, Harlow, Essex, UK, $214 \mathrm{p}$

Daie J., 1985. Carbohydrate partitioning and metabolism in crops. Hort. Rev., 7, 69-109.

Dale J. E., 1982. The growth of leaves. Studies in Biology n ${ }^{\circ} 137 . \mathrm{Ed}$. Edward Arnold, London, UK, 60 p.

Duffus C. M., Duffus J. H., 1984. Carbohydrate metabolism in plants. Longman, Harlow, Essex, UK. 183 p.

Gachon L., 1973. Influence du système cultural sur l'économie de l'azote en sol argilo-calcaire de Limagne. Sci. Sol, 3, 177-197.

Gosse G., Rollier M., Rode J. C., Chartier M., 1983. Vers une modélisation de la production chez le colza de printemps. In $\sigma^{\circ} \mathrm{Congr}$. Intern. Colza, Paris, mai 1983, 116-125.

Jourdan J., 1980. Variations saisonnières de la morphogenèse et de la croissance des systèmes aériens et souterrains chez le peuplier; ieurs relations avec les glucides et les transferts mineraux. Thèse de Docteur ès Sciences, USM, Grenoble, 160 p.

Léopold A. C., 1961. Senescence in plant development. Science, 134, $1727-1732$

Léopold A. C., 1975. Aging, senescence, and turnover in plants. Bioscience, 25, 659-662.

Makino A., Mae T., Ohira K., 1984a. Relation between nitrogen and ribulose-1,5-biphosphate carboxylase in rice leaves from emergence through senescence. Plant Cell Physiol., 25, 429-437.

Makino A., Mae T., Ohira K., 1984h. Changes in photosynthetic capacity in rice leaves from emergence through senescence. Analysis from ribulose-1, 5-biphosphate carboxylase and leaf conductance. Plant Cell Physiol., 25, 511-521.

Major D. J., Bole J. B., Charnetski W. A., 1980. The contribution of leaves to rapeseed yield. Canada Agriculture, 25, 11-13.

Malvoisin P., 1984. Organogenèse et croissance du maître-brin du blé tendre (Triticum aestivum) du semis à la floraison. I. Relations observées entre la croissance foliaire et la différenciation des ébauches foliaires ou florales. Agronomie, 4, 557-564.

Neales T. F., Incoll L. D., 1968. The control of leaf photosynthesis rate by the level of assimilate concentration in the leaf : a review of the hypothesis. Bot. Rev., 34, 107-123.
Netzer M. H., 1986. Recherches sur le déterminisme de la floraison de trois cultivars de Brassica napus $L$. var oleifera en conditions contrôlées. Thèse de Doctorat $3^{\circ}$ cycle, Univ. Pierre et Marie Curie, $100 \mathrm{p}$.

Netzer M. H., Tittonel E., Merrien A., 1986. Initiation florale du colza d'hiver Brassica napus L. cv Jet Neuf en conditions contrôlées. CETIOM, Inf. tech., 96, 3-9.

Pate J. S., Atkins C. A., 1983. Xylem and phloem transport and the functional economy of carbon and nitrogen of a legume leaf. Plant Physiol., 71, 835-840.

Pate J. S., Layzell D. B., 1981. Carbon and nitrogen partitioning in the whole plant - a thesis based on empirical modeling. 94-134. In : Bewley J.D., Nitrogen and carbon metabolism, Martinus Nijhoff, Dr W. Junk, 248 p.

Quilleré I., 1986. Quelques aspects de la dynamique des réserves en relation avec la croissance chez le colza d'hiver (Brassica napus $L$. var oleifera Metzg). Thèse de Docteur Ingénieur, ENSAIA Nancy, 134 p. + annexes.

Robelin M., Triboi A. M., 1983. Assimilation nette d'une culture dc colza d'hiver au cours d'un cycle de végétation sous l'influence de l'environnement climatique, de la densité du peuplement et de la fertilisation azotée. $6^{e}$ Congrès Intern. Colza, Paris mai 1983, 98-103.

Rood S. B., Major D. J., Carefoot J. M., Bole J. B., 1984. Seasonal distribution of nitrogen in oilseed rape. Field Crops Res., 8, 333-340.

Thimann K. V., 1980. The senescence of leaves. In Senescence in plants, K. V. Thimann (Ed.), CRC Press, Boca Raton, Fl., USA, 85-115.

Thomas H., Stoddart J. L., 1980. Leaf senescence. Annu. Rev. Plant Physiol., 31, 83-111.

Tittonel E. D., Desplantes G., Grangeret I., Pinochet X., 1982. Modifications morphologiques d'un bourgeon de colza (Brassica napus) au cours de la formation des ébauches florales. CETIOM, Inf. tech., 78, 15-24.

Tittonel E. D., 1987. Events concerning floral initiation at the beginning of spring. $7^{e}$ Congrès Intern. Colza, Poznan, mai 1987, 6 p.

Triboi A. M., 1984. Métabolisme azoté dans la plante. Trait Union agric. $89,16-20$.

Vann D. R., Fletcher J. S., Acchireddy N. R., Beevers L., 1986. Influence of partial defoliation of green pepper on the senescence, growth, and nitrate reductase of the remaining leaf. Plant Soil, 91, 357-361. 